\title{
Acceptance of the different denominations for reflex sympathetic dystrophy
}

\author{
B Alvarez-Lario, Í Aretxabala-Alcíbar, J Alegre-López, J L Alonso-Valdivielso
}

\begin{abstract}
Objective-To elucidate the real impact in the medical literature of the different denominations for reflex sympathetic dystrophy (RSD).

Methods-A search was performed through the Medline database (WinSPIRS, SilverPlatter International, NS), from 1995 to 1999 , including the following descriptors: RSD, complex regional pain syndrome (CRPS), CRPS type I, algodystrophy, Sudeck, shoulder-hand syndrome, transient osteoporosis, causalgia, and CRPS type II.

Results-The descriptor RSD was detected in 576 references, algodystrophy in 54, transient osteoporosis in 42, CRPS type $I$ in 24, Sudeck in 16, and shoulderhand syndrome in 11 . One hundred records were obtained for the descriptor causalgia and five for CRPS type II. The descriptor RSD was detected in the title of 262 references, algodystrophy in 29, transient osteoporosis in 29, CRPS type I in 15, Sudeck in 3, shoulder-hand syndrome in 5 , causalgia in 17, and CRPS type II in 3 references.

Conclusions-The new CRPS terminology has not effectively replaced the old one. RSD and causalgia are the most used denominations.
\end{abstract}

(Ann Rheum Dis 2001;60:77-79)

Reflex sympathetic dystrophy (RSD) is a complex of symptoms that includes severe pain, swelling, autonomic vasomotor dysfunction, and impaired mobility of the affected extremities. RSD has been given various names, depending on the precipitating factor, the country concerned, or the specialty treating the patient: reflex sympathetic dystrophy in English speaking, Sudeck's atrophy in German speaking, and algodystrophy in French speaking countries; causalgia after nerve injury; postinfartion sclerodactyly by cardiologists; Pourfour du Petit syndrome by anaesthetists; and peripheral trophoneurosis or BabinskyFroment sympathetic paralysis by neurologists. ${ }^{1}$ Post-traumatic osteoporosis, transient osteoporosis, algoneurodystrophy, shoulderhand syndrome, gardenalic rheumatism, neurotrophic rheumatism, and reflex neurovascular dystrophy have been other terms according to different countries or different medical specialties. $^{2}$

In 1994 a working group of the International Association for the Study of Pain (IASP) developed a consensus definition and proposed a new terminology. Thus the term complex regional pain syndrome (CRPS) type I replaces the name RSD, and the term CRSP type II, which requires demonstrable peripheral nerve injury, replaces the term causalgia. ${ }^{3}$ Five years after the introduction of this new terminology we have studied its real impact in the medical literature and the terms currently used to name this syndrome.

\section{Methods}

We detected the reports written between 1995 and 1999 that included the following descriptors: reflex sympathetic dystrophy, complex regional pain syndrome type I, complex regional pain syndrome type 1, algodystrophy, Sudeck, shoulder-hand syndrome, transient osteoporosis, causalgia, complex regional pain syndrome type II, complex regional pain syndrome type 2, complex regional pain syndrome, and CRPS. Other descriptors were refused by none or minimal use. The search was performed through Medline database (WinSPIRS 4.01, 2000 edition, updated until June 2000, SilverPlatter International, NS). The following options were registered for every reference: title, author(s), author's address, source, publication year, language of the article, country of publication, and medical subject heading major and minor.

\section{Results}

The descriptor "reflex sympathetic dystrophy" was detected in 576 references, "algodystrophy" in 54, "transient osteoporosis" in 42, "complex regional pain syndrome type I" in 24, "Sudeck" in 16, and "shoulder-hand syndrome" in 11 (table 1). One hundred records were obtained for the descriptor "causalgia" and five for the descriptor "complex regional pain syndrome type II". Some authors used the terms "type 1 and 2 " instead of "type I and II", and records using both terminologies can be found. The descriptor "complex regional pain syndrome", without type, detects more records than the combination of complex regional pain syndrome type I and type II (table 1); this might be caused by the trend for several authors to use the term complex regional pain syndrome merely as a synonym for RSD. The descriptor CRPS (abbreviated form and without type) was considered imprecise, because articles not related were obtained (cysteine-rich protein, C reactive protein, C receptor protein, coordinated research projects, cardiac rehabilitation programmes, collagen related peptides, etc).

When we considered the presence of the different terms in the title (table 1, parentheses), the descriptor "reflex sympathetic 
Table 1 References detected with the different descriptors (parenthetically, references with the descriptor in the title)

\begin{tabular}{|c|c|c|c|c|c|c|}
\hline & 1995 & 1996 & 1997 & 1998 & 1999 & Total \\
\hline Reflex sympathetic dystrophy & $118(65)$ & $107(46)$ & $137(57)$ & $129(57)$ & $85(37)$ & $576(262)$ \\
\hline Algodystrophy & $7(4)$ & $10(6)$ & $16(11)$ & $11(6)$ & $10(2)$ & $54(29)$ \\
\hline Transient osteoporosis & $9(7)$ & $8(5)$ & $8(7)$ & $9(5)$ & $8(5)$ & $42(29)$ \\
\hline CRPS ${ }^{\star}$ type I & $1(0)$ & $1(1)$ & $3(3)$ & $7(5)$ & $12(6)$ & $24(15)$ \\
\hline Sudeck atrophy & $2(0)$ & $4(1)$ & $7(1)$ & $2(1)$ & $1(0)$ & $16(3)$ \\
\hline Shoulder-hand syndrome & $2(2)$ & $2(0)$ & $3(0)$ & $1(0)$ & $3(3)$ & $11(5)$ \\
\hline Causalgia & $18(4)$ & $19(1)$ & $23(4)$ & $22(5)$ & $18(3)$ & $100(17)$ \\
\hline CRPS type II & $0(0)$ & $0(0)$ & $1(1)$ & $3(2)$ & $1(0)$ & $5(3)$ \\
\hline Complex regional pain syndrome & $4(2)$ & $5(4)$ & $16(15)$ & $19(15)$ & $28(23)$ & $72(59)$ \\
\hline
\end{tabular}

${ }^{\star}$ CRPS $=$ complex regional pain syndrome.

CRPS type I includes type I and type 1. CRSP type II includes type II and type 2.

Updated until June 2000. The database is continually updated and new references, mainly 1999 references, will be incorporated during the year 2000 .

dystrophy" was detected in 262 references, "algodystrophy" in 29, "transient osteoporosis" in 29 , "complex regional pain syndrome type I" in 15, "Sudeck" in 3, "shoulder-hand syndrome" in 5 , causalgia in 17 , and "complex regional pain syndrome type II" in 3 references.

The term RSD was used by authors from many countries, mainly English speaking authors (table 2), and from different medical specialties and journals (table 3). The term algodystrophy was used by European authors, especially French and Polish authors (table 2), who were mainly orthopaedic surgeons, rheumatologists, internists, and radiologists. The term transient osteoporosis was found in orthopaedic, radiology, and rheumatology journals, but not in pain and neurology journals (table 3 ). The term Sudeck atrophy was used only by European authors, especially German ones (table 2), who mainly were orthopaedic surgeons. The new terminology, CRPS types I and II, was found in papers written in English, mainly from the United States and Netherlands (table 2) and was especially used by pain and neurology

Table 2 Number of publications for each descriptor according to the author's country of origin

\begin{tabular}{lrrrrrrrrrrr} 
& USA & UK & Can & Ger & Fra & Neth & Belg & Spa & Pol & Ita & fap \\
\hline RSD & 251 & 87 & 14 & 36 & 31 & 30 & 30 & 15 & 13 & 9 & 8 \\
Algodystrophy & 5 & 7 & 0 & 3 & 15 & 0 & 5 & 3 & 11 & 3 & 0 \\
Transient osteoporosis & 16 & 4 & 1 & 6 & 2 & 0 & 4 & 0 & 0 & 3 & 1 \\
CRPS & 41 & 5 & 2 & 2 & 1 & 14 & 1 & 0 & 1 & 0 & 0 \\
Sudeck & 0 & 0 & 0 & 11 & 1 & 0 & 1 & 0 & 2 & 0 & 0 \\
Shoulder-hand syndrome & 2 & 1 & 0 & 0 & 0 & 0 & 0 & 1 & 3 & 1 & 0 \\
Causalgia & 48 & 15 & 2 & 4 & 1 & 10 & 1 & 2 & 2 & 0 & 2 \\
\hline
\end{tabular}

USA = United States; UK = United Kingdom; Can = Canada $;$ Ger = Germany; Fra = France; Neth $=$ Netherlands $;$ Belg $=$ Belgium $;$ Spa $=$ Spain $;$ Pol = Poland $;$ Ita = Italy $;$ Jap = Japan $;$ RSD = reflex sympathetic dystrophy; CRPS = complex regional pain syndrome.

Table 3 Number of publications for each descriptor according to the specialty of the journals retrieved

\begin{tabular}{lrrrrrrrrr}
\hline & Orthop & Pain & Neurol & Rheum & Rehabil & RX & Med & Surg & Others \\
\hline RSD & 143 & 91 & 75 & 37 & 28 & 35 & 64 & 15 & 88 \\
Algodystrophy & 27 & 0 & 2 & 4 & 0 & 5 & 8 & 1 & 7 \\
Transient osteoporosis & 15 & 0 & 0 & 7 & 1 & 11 & 2 & 0 & 6 \\
CRPS & 7 & 36 & 13 & 1 & 2 & 2 & 6 & 0 & 5 \\
Sudeck & 10 & 2 & 1 & 0 & 0 & 2 & 0 & 1 & 0 \\
Shoulder-hand syndrome & 1 & 0 & 3 & 0 & 1 & 0 & 2 & 0 & 4 \\
Causalgia & 17 & 20 & 27 & 1 & 2 & 0 & 13 & 5 & 15
\end{tabular}

Orthop = orthopaedic, hand surgery, sport medicine, and plastic surgery journals; Pain = pain and anaesthesiology journals; Neurol = neurology and neurosurgery journals; Rheum = rheumatology journals; Rehabil $=$ rehabilitation journals; $\mathrm{RX}=$ radiology and nuclear medicine journals; Med = internal and general medicine journals; Surg = general surgery journals; RSD = reflex sympathetic dystrophy; CRPS = complex regional pain syndrome. specialists in pain and neurology journals (table 3). Causalgia was also more used by English speaking and Dutch authors (table 2).

\section{Discussion}

Involvement of the sympathetic system seems unlikely in RSD. ${ }^{56}$ To replace this imprecise term the IASP proposed an "umbrella" terminology which does not make any reference to the cause of this entity. Although there is from 1995 up to 1999 a constant increase in its use, the new terminology has not been extensively accepted in the medical literature. Our study shows that the old terms are much more used than the new ones. Overall, there have not been important changes and the most used term is "reflex sympathetic dystrophy".

It is difficult to change the name of an entity which has been widely used. Although the old nomenclature does not define the syndrome well, there are historical reasons to keep up these old names. A good reason for the change might be that the new terminology and the "draft criteria" are much more precise, but this does not seem to be the case as the term CRPS is too vague. ${ }^{78}$ Alternative explanations for the infrequent use of CRPS might be the fact that some authors were not aware of the conclusions of the working group of the IASP and of the existence of this new terminology, which was published in pain journals and not in orthopaedic or rheumatology journals; and the fact that authors, reviewers, or editors were reluctant to substitute CRPS for older terminologies because they feared that most readers of these journals were unaware its meaning.

A clear contribution of the new terminology is the definition of CRPS type II (causalgia), which requires a peripheral nerve injury; however, several authors who used the new term in their papers did not discriminate between the two types and used CRPS terminology, without type, as a synonym for RSD.

We conclude that the new terminology has not effectively replaced the old. RSD and causalgia are still the most commonly used denominations. The CRPS terminology is mainly used in pain and neurology journals. A more extensive international consensus could be useful to unify terminology for these common disorders. 
1 Veldman PHJM, Reynen HM, Arntz IE, Goris RJA. Signs and symptoms of reflex sympathetic dystrophy: prospective study of 829 patients. Lancet 1993;342:1012-16.

2 Doury P, Dequeker J. Algodystrophy/reflex sympathetic dystrophy syndrome. In: Klippel JH, Dieppe PA, eds. Rheumatology. London: Mosby, 1994:7.38.1-8.

3 Merskey H, Bogduck N. Classification of chronic pain: descriptions of chronic pain syndromes and definition of pain terms. 2nd ed. Seattle, Washington: IASP Press, 1994:40-3.

4 Stanton-Hicks M, Jänig W, Hassenbusch S, Haddox JD, Boas R, Wilson P. Reflex sympathetic dystrophy: changing concepts and taxonomy. Pain 1995;63:127-33.

5 Schott GD. An unsympathetic view of pain. Lancet 1995;345:634-6.
6 Berthelot JM, Glemarec J, Guillot P, Maugars Y, Prost A. Algodystrophy (reflex sympathetic dystrophy) and causalgia: novel concepts regarding the nosology, pathophysiology, and pathogenesis of complex regional pain syndromes. Is the sympathetic hyperactivity hypothesis wrong? Rev Rhum Engl Ed 1997;64:481-91.

7 Galer BS, Bruehl S, Harden RN. IASP diagnostic criteria for complex regional pain syndrome: a preliminary empirical validation study. International Association for the Study of Pain. Clin J Pain 1998;14:48-54.

8 Harden RN, Bruehl S, Galer BS, Saltz S, Bertram M, Backonja $M$, et al. Complex regional pain syndrome: are the sive? Pain 1999;83:211-19. 